Volume 134, Number 3, Pages 737-747

S 0002-9939(05)08016-0

Article electronically published on July 18, 2005

\title{
JOINT MEASURABILITY AND \\ THE ONE-WAY FUBINI PROPERTY FOR A CONTINUUM OF INDEPENDENT RANDOM VARIABLES
}

\author{
PETER J. HAMMOND AND YENENG SUN
}

(Communicated by Carl G. Jockusch, Jr.)

\begin{abstract}
As is well known, a continuous parameter process with mutually independent random variables is not jointly measurable in the usual sense. This paper proposes an extension of the usual product measure-theoretic framework, using a natural "one-way Fubini" property. When the random variables are independent even in a very weak sense, this property guarantees joint measurability and defines a unique measure on a suitable minimal $\sigma$-algebra. However, a further extension to satisfy the usual (two-way) Fubini property, as in the case of Loeb product measures, may not be possible in general. Some applications are also given.
\end{abstract}

\section{INTRODUCTION}

In [7] (p. 102), Doob made the claim that processes with mutually independent random variables are only useful in the discrete parameter case. There are indeed essential measurability difficulties associated with a continuous parameter process with random variables that are independent even in a weak sense.

Two kinds of measurability problem usually arise. The first concerns joint measurability; namely, except in some trivial cases, such a process can never be jointly measurable with respect to the completion of the usual product $\sigma$-algebra on the joint space of parameters and sample points. This means that the conditions of independence and joint measurability in the usual sense are incompatible with each other. Thus, one cannot integrate the process or take its distribution as a function on the joint space.

The second problem concerns sample measurability; as noted in [6], with further elaborations in [10], the set of sample points whose corresponding sample functions are not Lebesgue measurable has outer measure one, so Lebesgue measure offers no basis for a meaningful concept of the mean or the distribution of a sample function. Nevertheless, it has been hypothesized in a vast literature in economics that an exact version of the law of large number holds for such a process, which means that

Received by the editors July 31, 2002 and, in revised form, October 8, 2004.

2000 Mathematics Subject Classification. Primary 28A05, 60G07; Secondary 03E20, 03H05, $28 \mathrm{~A} 20$.

Key words and phrases. Loeb product measures, product-measurable sets, continuum of independent random variables, joint measurability problem, one-way Fubini property.

Part of this work was done when the first author was visiting Singapore in November 1999 and when the second author was visiting Stanford in July 2002.

(C)2005 American Mathematical Society Reverts to public domain 28 years from publication 
the observable mean or distribution of a sample function is essentially independent of the particular sample realization (see [2, 9], and [17] for some of the references).

In [16]-19], the Loeb product framework introduced in [12] (see also [14]) is used to formulate and prove various versions of the exact law of large numbers and their converses. The key point is that the Loeb product extends the usual measuretheoretic product, as noted by Anderson in 11, while retaining the common Fubini property (as first shown by Keisler in [11] - see also [13] and 14]). It also accommodates an abundance of nontrivial independent processes (Theorem 6.2 in [17). In particular, Keisler's Fubini theorem implies that both the sample and joint measurability problems are automatically solved for independent processes that are Loeb product measurable.

The approach used in [16]-19] works with a given "nice" extension of the usual measure-theoretic product, which happens to allow many nontrivial independent processes that are jointly measurable. An important question in the other direction is whether, given a particular nontrivial independent process, there exists a "nice" extension of the usual measure-theoretic product that makes this process jointly measurable. As noted in Remark 3.2 below, there may be no such extension satisfying the Fubini property. Nevertheless, this paper shows that the joint measurability problem for such a process can indeed be solved by considering a natural probability space we call the "one-way Fubini" extension (see Definition 2.3).

The rest of the paper is organized as follows. Section 2 presents some basic definitions. The main result (Theorem 3.1) concerning the unique extension is presented in Section 3. Section 4 includes some applications of the main result. Finally, the proof of Theorem 3.1 is given in Section 5 .

\section{BASIC DEFINITIONS}

Let $(T, \mathcal{T}, \lambda)$ be a probability space which is to be used as a parameter space for a process. If one likes, $T$ can be taken to be the unit interval $[0,1]$, but $(T, \mathcal{T}, \lambda)$ is not restricted to be the Lebesgue measure structure.

Let $(\Omega, \mathcal{A}, P)$ be a sample probability space. For example, it can be the product of a continuum of copies of some other basic probability space (the existence of such a continuum product is based on the Axiom of Choice and Kolmogorov's consistency theorem), or some extension of this product, or some other space entirely. As usual in probability theory, it is not necessary to specify precisely the sample probability space, provided some general existence issues are resolved.

Let $(T \times \Omega, \mathcal{T} \otimes \mathcal{A}, \lambda \times P)$ be the usual product probability space (see, for example, [15]). Let $g$ be a process from $T \times \Omega$ to some Polish space $X$ with Borel $\sigma$-algebra $\mathcal{B}$.

We make the following assumptions on $g$ :

(1) For $\lambda$-almost all $t \in T, g_{t}$ is a random variable defined on $\Omega$ whose distribution $P g_{t}^{-1}$ on $X$ is denoted by $\mu_{t}$.

(2) For every $B \in \mathcal{B}$, the mapping $t \mapsto \mu_{t}(B)$ is $\mathcal{T}$-measurable.

(3) The random variables $g_{t}$ are almost surely pairwise independent in the sense that for $\lambda$-almost all $t_{1} \in T, g_{t_{1}}$ is independent of $g_{t_{2}}$ for $\lambda$-almost all $t_{2} \in T$.

As noted in Lemma 1 of [9], the second condition is equivalent to the measurability of the distribution mapping $t \mapsto \mu_{t}$ from $T$ to $\mathcal{M}(X, \mathcal{B})$, where the space $\mathcal{M}(X, \mathcal{B})$ of distributions on $(X, \mathcal{B})$ is given the weak convergence topology and associated Borel $\sigma$-algebra. The third condition is an idealized version of weak dependence in probability theory (see the discussion in [18, p. 437). When $\lambda$ is atomless, this 
condition is weaker than mutual independence. One may simply observe that if the random variables $g_{t}$ are mutually independent, then they are pairwise independent and hence also almost surely pairwise independent. When $\lambda$ has an atom $A$, then the third condition implies that for almost all $t \in A, g_{t}$ is independent of itself and hence almost surely a constant. Note that $g$ is not $\mathcal{T} \otimes \mathcal{A}$-measurable except in the trivial case when, for almost all $t \in T, g_{t}$ is a.s. a constant (see [7, [18, and also Corollary 4.1 below).

Let $(T, \mathcal{T}, \lambda)$ and $(\Omega, \mathcal{A}, P)$ be probability spaces, and $(T \times \Omega, \mathcal{T} \otimes \mathcal{A}, \lambda \times P)$ the usual product probability space. Below are the formal definitions of the various Fubini properties used in this paper.

Definition 2.1. (1) A probability space $(T \times \Omega, \mathcal{W}, Q)$ that extends $(T \times \Omega, \mathcal{T} \otimes$ $\mathcal{A}, \lambda \times P)$ is said to be a Fubini extension if, for any real-valued $\mathcal{W}$-integrable function $f$, the two functions $f_{t}$ and $f_{\omega}$ are integrable respectively on $(\Omega, \mathcal{A}, P)$ for $\lambda$-almost all $t \in T$ and on $(T, \mathcal{T}, \lambda)$ for $P$-almost all $\omega \in \Omega$; moreover, $\int_{\Omega} f_{t} d P$ and $\int_{T} f_{\omega} d P$ are integrable respectively on $(T, \mathcal{T}, \lambda)$ and on $(\Omega, \mathcal{A}, P)$, with $\int_{T \times \Omega} f d Q=$ $\int_{T}\left(\int_{\Omega} f_{t} d P\right) d \lambda=\int_{\Omega}\left(\int_{T} f_{\omega} d \lambda\right) d P$.

(2) The probability space $(T \times \Omega, \mathcal{W}, Q)$ is said to be a generalized Fubini extension of $(T \times \Omega, \mathcal{T} \otimes \mathcal{A}, \lambda \times P)$ if there exist extensions $\left(T, \mathcal{T}^{\prime}, \lambda^{\prime}\right)$ and $\left(\Omega, \mathcal{A}^{\prime}, P^{\prime}\right)$ of $(T, \mathcal{T}, \lambda)$ and $(\Omega, \mathcal{A}, P)$ respectively such that $(T \times \Omega, \mathcal{W}, Q)$ is a Fubini extension of $\left(T \times \Omega, \mathcal{T}^{\prime} \otimes \mathcal{A}^{\prime}, \lambda^{\prime} \times P^{\prime}\right)$.

Given the process $g$, suppose that $(T \times \Omega, \mathcal{T} \otimes \mathcal{A}, \lambda \times P)$ has a generalized Fubini extension $(T \times \Omega, \mathcal{W}, Q)$ such that $g$ is $\mathcal{W}$-measurable. In this extended framework, the joint measurability problem has obviously been solved, as has the sample measurability problem because $g_{\omega}$ is $\mathcal{T}^{\prime}$-measurable.

The second definition is needed because the existence of a generalized Fubini extension does not imply the existence of a Fubini extension that makes $g$ measurable. The following example shows this.

Example 2.2. Take a sequence $\left\{\beta_{n}\right\}_{n \in \mathbb{N}}$ of iid random variables defined on a probability space $(\Lambda, \mathcal{G}, \mu)$ and having a uniform distribution on $[0,1]$. Then transfer this sequence to $\left\{{ }^{*} \beta_{n}\right\}_{n \in N_{N}}$ in the usual non-standard model, where ${ }^{*} \mathbb{N}$ denotes the set of hyper-integers (see [14]).

Let $m$ be an infinitely large hyper-integer, and $T$ the set $\{1,2, \ldots, m\}$. Then let $(T, \mathcal{T}, \lambda)$ be the Loeb counting probability space on $T$. Finally, let $(\Omega, \mathcal{A}, P)$ be the Loeb space of the transfer of $(\Lambda, \mathcal{G}, \mu)$, and define the random variable $b_{t}={ }^{\circ}\left({ }^{*} \beta_{t}\right)$ for each $t \in T$.

This construction yields a pair of Loeb probability spaces $(T, \mathcal{T}, \lambda)$ and $(\Omega, \mathcal{A}, P)$ with the following properties (see also [2, p. 2200): (1) there is a jointly measurable process $b$ on the Loeb product $(T \times \Omega, \mathcal{T} \otimes \mathcal{A}, \lambda \otimes P)$, which is the Loeb space of the internal product $\left(T \times{ }^{*} \Lambda, \mathcal{T} \otimes{ }^{*} \mathcal{G}, \lambda \times{ }^{*} \mu\right)$; (2) the random variables $b_{t}$ are pairwise independent; (3) for each $t \in T, b_{t}$ has uniform distribution on [0,1]. By Theorem 5.2 and Proposition 7.16 in [17, the sample functions $b_{\omega}$ are almost surely pairwise independent and also almost surely have an identical uniform distribution on $[0,1]$. Take any one $\omega_{0} \in \Omega$ such that $b_{\omega_{0}}$ is uniformly distributed on $[0,1]$ and independent of $b_{\omega}$ for $P$-almost all $\omega \in \Omega$. Let $\mathcal{T}_{0}$ be the $\sigma$-algebra generated by $b_{\omega_{0}}$. Obviously, $\mathcal{T}_{0} \subseteq \mathcal{T}$. Let $\lambda_{0}$ be the restriction of $\lambda$ to $\mathcal{T}_{0}$. Then the process $b$ does satisfy assumptions $1-3$ restated for the product probability space $\left(T \times \Omega, \mathcal{T}_{0} \otimes \mathcal{A}, \lambda_{0} \times P\right)$. Furthermore, the Loeb product space $(T \times \Omega, \mathcal{T} \otimes \mathcal{A}, \lambda \otimes P)$ is a generalized Fubini 
extension of $\left(T \times \Omega, \mathcal{T}_{0} \otimes \mathcal{A}, \lambda_{0} \times P\right)$. Nevertheless, the latter space has no Fubini extension that makes $b$ jointly measurable. Indeed, for it to have such an extension, $b_{\omega}$ would have to be $\mathcal{T}_{0}$-measurable for $P$-almost all $\omega \in \Omega$. Yet this is impossible given the fact that, for $P$-almost all $\omega \in \Omega$, the random variables $b_{\omega_{0}}$ and $b_{\omega}$ are independent and uniformly distributed on $[0,1]$.

Even though a generalized Fubini extension is weaker, it may still not exist in general. Indeed, Remark 3.2 below shows that for any given atomless parameter space $(T, \mathcal{T}, \lambda)$, such an extension does not exist at all for an extended sample space based on the general product measure space, as discussed in [4] (p. 230). This failure motivates the following analogous definition.

Definition 2.3. A probability space $(T \times \Omega, \mathcal{W}, Q)$ that extends $(T \times \Omega, \mathcal{T} \otimes \mathcal{A}, \lambda \times P)$ is said to be a one-way Fubini extension if, given any real-valued $\mathcal{W}$-integrable function $f$, the function $f_{t}$ is integrable on $(\Omega, \mathcal{A}, P)$ for $\lambda$-almost all $t \in T$, and moreover $\int_{\Omega} f_{t} d P$ is integrable on $(T, \mathcal{T}, \lambda)$, with $\int_{T \times \Omega} f d Q=\int_{T}\left(\int_{\Omega} f_{t} d P\right) d \lambda$.

The purpose of this paper is to show how, for any process $g$ satisfying assumptions (1)-(3), the joint measurability problem can be solved by using the one-way Fubini extension defined above. In particular, if $\mathcal{F}$ is the minimal extension of the usual product $\sigma$-algebra $\mathcal{T} \otimes \mathcal{A}$ such that $g$ is $\mathcal{F}$-measurable, then there is a unique probability measure $\nu$ on $\mathcal{F}$ such that $(T \times \Omega, \mathcal{F}, \nu)$ is a one-way Fubini extension of the product space $(T \times \Omega, \mathcal{T} \otimes \mathcal{A}, \lambda \times P)$. Moreover, if $g$ is measurable in any one-way Fubini extension (or in any generalized one-way Fubini extension defined analogously to part (2) of Definition 2.1), then that extension is simply a further extension of the minimal one-way Fubini extension $(T \times \Omega, \mathcal{F}, \nu)$ of the same product space. Unlike in the usual (two-way) Fubini setting, nothing is really gained by considering a generalized extension.

Although the one-way Fubini extension makes the process $g$ jointly measurable, it is in general false that the sample function $g_{\omega}$ is $\mathcal{T}$-measurable for $P$-almost all $\omega \in \Omega$. So the reverse order of integration in $(T \times \Omega, \mathcal{F}, \nu)$ is meaningless. Of course, the Fubini property idealizes the usual rules governing double or iterated sums in the discrete setting, so it should be imposed whenever possible. From another point of view, the one-way Fubini property ensures that the extended measure $\nu$ on $\mathcal{F}$ takes the correct values. Otherwise, as noted in Remark 4.4 below, one may obtain completely arbitrary and meaningless extensions.

We are now ready to state the main theorem.

\section{THE MAIN THEOREM}

Let $g$ be the process defined in Section 2. Define the mapping $H: T \times \Omega \rightarrow$ $T \times \Omega \times X$ by $H(t, \omega):=(t, \omega, g(t, \omega))$. Note that for each $t \in T$, the mapping $H_{t}: \Omega \rightarrow \Omega \times X$ satisfies $H_{t}(\omega)=\left(\omega, g_{t}(\omega)\right)$.

Let $\mathcal{E}:=\mathcal{T} \otimes \mathcal{A} \otimes \mathcal{B}$ denote the product $\sigma$-algebra on $T \times \Omega \times X$. Let $\mathcal{F}:=$ $\left\{H^{-1}(E): E \in \mathcal{E}\right\}$. Then it is clear that $\mathcal{F}$ is a $\sigma$-algebra. Also, the first two components of $H(t, \omega)$ are given by the identity mapping $\operatorname{id}_{T \times \Omega}$ on $T \times \Omega$, while the last component is $g(t, \omega)$. Hence, $\mathcal{F}$ is the smallest $\sigma$-algebra such that $\operatorname{id}_{T \times \Omega}$ and $g$ are both measurable. This means that $\mathcal{F}$ is the smallest extension of the product $\sigma$-algebra $\mathcal{T} \otimes \mathcal{A}$ such that $g$ is measurable.

The following theorem shows that, under the assumptions (1)-(3) in Section 2 , there is a unique probability measure $\nu$ on $\mathcal{F}$ which extends $\lambda \times P$ on $\mathcal{T} \otimes \mathcal{A}$ and 
has the property that integrating a $\nu$-integrable function $f$ on $T \times \Omega$ is equivalent to evaluating an iterated double integral in the particular order $\int_{T}\left(\int_{\Omega} f_{t} d P\right) d \lambda$; i.e., $(T \times \Omega, \mathcal{F}, \nu)$ is a one-way Fubini extension of $(T \times \Omega, \mathcal{T} \otimes \mathcal{A}, \lambda \times P)$. Since $\mathcal{F}$ is the smallest extension of the product $\sigma$-algebra $\mathcal{T} \otimes \mathcal{A}$ such that $g$ is measurable, the measure space $(T \times \Omega, \mathcal{F}, \nu)$ may not be complete. On the other hand, one can easily see that the completion of $(T \times \Omega, \mathcal{F}, \nu)$ is still a one-way Fubini extension of $(T \times \Omega, \mathcal{T} \otimes \mathcal{A}, \lambda \times P)$.

Theorem 3.1. Suppose assumptions (1)-(3) in Section 2 are satisfied.

(1) For any $E \in \mathcal{E}=\mathcal{T} \otimes \mathcal{A} \otimes \mathcal{B}$, for $\lambda$-a.e. $t \in T$ the set $H_{t}^{-1}\left(E_{t}\right)$ is $\mathcal{A}$ measurable, with $P\left(H_{t}^{-1}\left(E_{t}\right)\right)=\left(P \times \mu_{t}\right)\left(E_{t}\right)$; also, the mapping $t \mapsto$ $\left(P \times \mu_{t}\right)\left(E_{t}\right)$ is $\lambda$-integrable.

(2) There is a unique probability measure $\nu$ on the measurable space $(T \times \Omega, \mathcal{F})$ such that for any $F \in \mathcal{F}$, the set $F_{t}$ is $\mathcal{A}$-measurable for $\lambda$-almost all $t \in T$, and $t \mapsto P\left(F_{t}\right)$ is a $\lambda$-integrable function with $\nu(F)=\int_{T} P\left(F_{t}\right) d \lambda$.

(3) $(T \times \Omega, \mathcal{F}, \nu)$ is a one-way Fubini extension of $(T \times \Omega, \mathcal{T} \otimes \mathcal{A}, \lambda \times P)$; moreover, $\nu$ is the unique extension of $\lambda \times P$ on $\mathcal{F}$ with this property.

Remark 3.2. Let $(T, \mathcal{T}, \lambda)$ be any atomless probability space, and $\mu$ any probability distribution on $\mathbb{R}$. Let $(\Omega, \mathcal{A}, P)$ be the continuum product probability space $(\mathbb{R}, \mathcal{B}, \mu)^{T}$ (see [4, p. 230). Let $f: T \times \Omega \rightarrow \mathbb{R}$ be an iid process obtained from the coordinate functions $f_{t}(\omega)=\omega(t)$ on the sample space $\Omega=\mathbb{R}^{T}$. Based on the simple idea used in [6] (see also [10]), it is pointed out in [19] that, for any given real-valued function $h$ on $T$, the collection $M_{h}$ of those sample functions that differ from $h$ at countably many points in $T$ has $P$-outer measure one (see Remark 6.3 in [19). Following a standard procedure (see, for example, 7], p. 69) one can extend the measure $P$ to a new measure $\bar{P}$ on the $\sigma$-algebra $\overline{\mathcal{A}}$ generated by $\mathcal{A} \cup\left\{M_{h}\right\}$ so that $\bar{P}\left(M_{h}\right)=1$. Thus, one establishes the absurd claim that almost all sample functions are essentially equal to an arbitrarily given function $h$.

Now assume that the common mean of the random variables $f_{t}$ is $m$. Take $h$ to be any function whose mean $a=\int_{T} h d \lambda$ is not equal to $m$. Since $f_{\omega}$ is $h$ for $\bar{P}$ -

almost all $\omega \in \Omega$, the sample mean $\int_{T} f_{\omega} d \lambda=a$ for $\bar{P}$-almost all $\omega \in \Omega$, and hence $\int_{\Omega}\left[\int_{T} f(t, \omega) d \lambda\right] d \bar{P}=a$. On the other hand, $\int_{T} \int_{\Omega}[f(t, \omega) d \bar{P}] d \lambda=\int_{T} m d \lambda=m$. This means that the two iterated integrals are different; i.e., $\int_{T}\left[\int_{\Omega} f(t, \omega) d \bar{P}\right] d \lambda \neq$ $\int_{\Omega}\left[\int_{T} f(t, \omega) d \lambda\right] d \bar{P}$.

This shows that the product space $(T \times \Omega, \mathcal{T} \otimes \overline{\mathcal{A}}, \lambda \times \bar{P})$ does not have even a generalized Fubini extension $(T \times \Omega, \mathcal{W}, Q)$ such that $f$ is $\mathcal{W}$-measurable; otherwise, the two iterated integrals would be the same. However, the product space does have a one-way Fubini extension, as shown in Theorem 3.1.

\section{Some APPLiCATions OF THE MAIN THEOREM}

The following proposition shows that, for any $\mathcal{T} \otimes \mathcal{A}$-measurable function $h$, the two random variables $g_{t}$ and $h_{t}$ are independent for $\lambda$-almost all $t \in T$. This means that any $\mathcal{T} \otimes \mathcal{A}$-measurable function differs fundamentally from the process $g$.

Proposition 4.1. Let $h$ be a measurable function from the product space $(T \times \Omega, \mathcal{T} \otimes \mathcal{A}, \lambda \times P)$ to a Polish space $Y$. Then, for $\lambda$-almost all $t \in T, g_{t}$ and $h_{t}$ are independent; i.e., $P\left(h_{t}^{-1}(D) \cap g_{t}^{-1}(B)\right)=P\left(h_{t}^{-1}(D)\right) P\left(g_{t}^{-1}(B)\right)$ for all Borel sets $B$ in $X$ and $D$ in $Y$. 
Proof. Let $E:=h^{-1}(D) \times B \in \mathcal{T} \otimes \mathcal{A} \otimes \mathcal{B}=\mathcal{E}$. Then $E_{t}=h_{t}^{-1}(D) \times B$ and $H_{t}^{-1}\left(E_{t}\right)=h_{t}^{-1}(D) \cap g_{t}^{-1}(B)$. So for $\lambda$-a.e. $t \in T$, part (1) of Theorem 3.1 implies that

$$
\begin{aligned}
P\left(h_{t}^{-1}(D) \cap g_{t}^{-1}(B)\right) & =P\left(H_{t}^{-1}\left(E_{t}\right)\right)=\left(P \times \mu_{t}\right)\left(E_{t}\right)=P\left(h_{t}^{-1}(D)\right) \mu_{t}(B) \\
& =P\left(h_{t}^{-1}(D)\right) P\left(g_{t}^{-1}(B)\right) .
\end{aligned}
$$

Now we can use an argument such as that in the proof of Theorem 7.6 in [17. There exist countable open bases $\mathcal{B}_{X}$ and $\mathcal{B}_{Y}$ for the respective topologies of the Polish spaces $X$ and $Y$ such that each base is closed under finite intersections. Because $\mathcal{B}_{X}$ and $\mathcal{B}_{Y}$ are countable, the above paragraph implies that there exists a $\lambda$-null set $S_{0} \subset T$ such that, for all $t \notin S_{0}$,

$$
P\left(g_{t}^{-1}\left(O_{X}\right) \cap h_{t}^{-1}\left(O_{Y}\right)\right)=P\left(g_{t}^{-1}\left(O_{X}\right)\right) \cdot P\left(h_{t}^{-1}\left(O_{Y}\right)\right)
$$

holds simultaneously for all $O_{X} \in \mathcal{B}_{X}$ and all $O_{Y} \in \mathcal{B}_{Y}$. Thus, for any $t \notin S_{0}$, the joint distribution $P\left(g_{t}, h_{t}\right)^{-1}$ on $X \times Y$ agrees with the product $P g_{t}^{-1} \times P h_{t}^{-1}$ of its marginals on the $\pi$-system $\left\{O_{X} \times O_{Y}: O_{X} \in \mathcal{B}_{X}, O_{Y} \in \mathcal{B}_{Y}\right\}$ for the $\sigma$-algebra $\mathcal{B}_{X} \otimes \mathcal{B}_{Y}$ on $X \times Y$. So by a result on the unique extension of measures (see 8 , p. 402), $P\left(g_{t}, h_{t}\right)^{-1}=P g_{t}^{-1} \times P h_{t}^{-1}$ on the whole product $\sigma$-algebra. This implies that $h_{t}$ and $g_{t}$ are independent for all $t \notin S_{0}$, which completes the proof.

The following obvious corollary was Proposition 1 in [18. When $\lambda$ is Lebesgue measure and the process $g$ is iid, a similar result was already noted by Doob in 7 ] (p. 67).

Corollary 4.2. If $g$ is measurable on $(T \times \Omega, \mathcal{T} \otimes \mathcal{A}, \lambda \times P)$, then for $\lambda$-almost all $t \in T$, the random variable $g_{t}$ is essentially constant.

Proof. Proposition 4.1 implies that for $\lambda$-a.e. $t \in T, g_{t}$ is independent of itself and hence a constant.

The following result extends Theorem 4.2 in the Loeb product framework of [3] to the general case.

Proposition 4.3. Let $C$ be a subset of $T \times \Omega$ such that $0<P\left(C_{t}\right)<1$ for $\lambda$-almost all $t \in T$. Suppose the events $C_{t}(t \in T)$ are almost surely pairwise independent; i.e., for $\lambda$-almost all $t_{1} \in T, C_{t_{1}}$ is independent of $C_{t_{2}}$ for $\lambda$-almost all $t_{2} \in T$. Then $C$ has outer measure one and inner measure zero with respect to $\lambda \times P$.

Proof. Let $g$ be the indicator function $1_{C}$ of $C$. Then $g$ is a process satisfying the assumptions in Section 1. Also, the random variables $g_{t}$ are almost surely pairwise independent. Take any $D \in \mathcal{T} \otimes \mathcal{A}$ and let $h$ be $1_{D}$. Proposition 4.1 implies that, for $\lambda$-almost all $t \in T$, the random variables $g_{t}$ and $h_{t}$ are independent. So therefore are the events $C_{t}$ and $D_{t}$; i.e., $P\left(C_{t} \cap D_{t}\right)=P\left(C_{t}\right) P\left(D_{t}\right)$.

Thus, if $D \subseteq C$, then for $\lambda$-almost all $t \in T, P\left(D_{t}\right)=P\left(C_{t}\right) P\left(D_{t}\right)$. Since $P\left(C_{t}\right)<1$ for $\lambda$-almost all $t \in T$, it follows that $P\left(D_{t}\right)=0$ for $\lambda$-almost all $t \in T$. By the Fubini theorem, because $D$ is an arbitrary $\mathcal{T} \otimes \mathcal{A}$-measurable subset of $C$, it follows that the inner measure $(\lambda \times P)_{*}(C)=0$.

On the other hand, if $C \subseteq D$, then for $\lambda$-almost all $t \in T, P\left(C_{t}\right)=P\left(C_{t}\right) P\left(D_{t}\right)$. Since $P\left(C_{t}\right)>0$ for $\lambda$-almost all $t \in T$, it follows that $P\left(D_{t}\right)=1$ for $\lambda$-almost all $t \in T$. By the Fubini theorem, $(\lambda \times P)(D)=1$. Because $D \supseteq C$ is arbitrary in $\mathcal{T} \otimes \mathcal{A}$, it follows that the outer measure $(\lambda \times P)^{*}(C)=1$. 
Remark 4.4. Suppose the set $C \subseteq T \times \Omega$ and the probability space $(\Omega, \mathcal{A}, P)$ are such that the events $C_{t}(t \in T)$ are almost surely pairwise independent with identical probability $p$, for some $0<p<1$. Let $g=1_{C}$, and let

$$
\mathcal{F}=\left\{\left(D_{1} \cap C\right) \cup\left(D_{2} \backslash C\right): D_{1}, D_{2} \in \mathcal{T} \otimes \mathcal{A}\right\}
$$

be the smallest extension of the product $\sigma$-algebra such that $g$ is measurable. If we require the one-way Fubini property on $(T \times \Omega, \mathcal{F})$, then the measure for $C$ must be

$$
\nu(C)=\int_{T}\left(\int_{\Omega} 1_{C} d P\right) d \lambda=\int_{T} P\left(C_{t}\right) d \lambda=p .
$$

On the other hand, if we do not require the one-way Fubini property, then for an arbitrarily given number $r \in[0,1]$, we can use the common procedure for extending measures (see, for example, [7, p. 69) to define

$$
\sigma_{r}\left(\left(D_{1} \cap C\right) \cup\left(D_{2} \backslash C\right)\right)=r(\lambda \times P)\left(D_{1}\right)+(1-r)(\lambda \times P)\left(D_{2}\right)
$$

for any $D_{1}, D_{2} \in \mathcal{T} \otimes \mathcal{A}$. Because of Proposition 4.3, this must be a well-defined probability measure on $(T \times \Omega, \mathcal{F})$, with $\sigma_{r}(C)=r$. So the one-way Fubini property allows us to select the "correct" measure for the extension and to ignore other completely meaningless extensions such as $\sigma_{r}$ for any $r \neq p$.

\section{Proof of the MAIN Theorem}

Lemma 5.1. Suppose that the random variables $f_{t}(t \in T)$ are all square-integrable and are almost surely uncorrelated; i.e., suppose each $f_{t} \in L_{2}(\Omega, \mathcal{A}, P)$ and, for a.e. $t_{1} \in T, E\left(f_{t_{1}} f_{t_{2}}\right)=E f_{t_{1}} \cdot E f_{t_{2}}$ for a.e. $t_{2} \in T$. Then, for every $A \in \mathcal{A}$, $\int_{A} f_{t} d P=P(A) E f_{t}$ for $\lambda$-a.e. $t \in T$.

Proof. Let $T^{\prime}$ be the set of all $t^{\prime} \in T$ such that the random variables $f_{t^{\prime}}$ and $f_{t}$ are uncorrelated for $\lambda$-a.e. $t \in T$. By hypothesis, $\lambda\left(T^{\prime}\right)=1$.

Following a standard procedure for Hilbert spaces, let $L$ be the smallest closed linear subspace of $L_{2}(\Omega, \mathcal{A}, P)$ containing both the family $\left\{f_{t}: t \in T^{\prime}\right\}$ and the constant function $1=1_{\Omega}$. Let $h$ be the orthogonal projection of the indicator function $1_{A}$ onto $L$, with $h^{\perp}$ as its orthogonal complement. Then $1_{A}=h+h^{\perp}$ where $E\left(h^{\perp} f_{t}\right)=\int_{\Omega} h^{\perp} f_{t} d P=0$ for all $t \in T^{\prime}$, and also $E h^{\perp}=\int_{\Omega} h^{\perp} d P=0$. It follows that $E\left(1_{A} f_{t}\right)=E\left(h f_{t}\right)$ for all $t \in T^{\prime}$, and also $E 1_{A}=P(A)=E h$.

Next, because $h \in L$, there exists a sequence of functions

$$
h_{n}=r_{n}+\sum_{k=1}^{i_{n}} \alpha_{n}^{k} f_{t_{n}^{k}}(n=1,2, \ldots)
$$

with $t_{n}^{k} \in T^{\prime}$, as well as $r_{n}$ and $\alpha_{n}^{k}\left(k=1, \ldots, i_{n}\right)$ all real, such that $h_{n} \rightarrow h$ in $L_{2}(\Omega, \mathcal{A}, P)$.

Let $T_{n}^{k}:=\left\{t \in T: f_{t}\right.$ and $f_{t_{n}^{k}}$ are uncorrelated $\}$. By hypothesis, $\lambda\left(T_{n}^{k}\right)=1$ because each $t_{n}^{k} \in T^{\prime}$. Define $T^{*}:=T^{\prime} \cap\left(\bigcap_{n=1}^{\infty} \bigcap_{k=1}^{i_{n}} T_{n}^{k}\right)$. Then $\lambda\left(T^{*}\right)=1$, because $\lambda\left(T^{\prime}\right)=1$. Also, for any $t \in T^{*}$, because $f_{t}$ and each $f_{t_{n}^{k}}$ are uncorrelated, one has

$$
E\left(h_{n} f_{t}\right)=r_{n} E f_{t}+\sum_{k=1}^{i_{n}} \alpha_{n}^{k} E\left(f_{t_{n}^{k}} f_{t}\right)=r_{n} E f_{t}+\sum_{k=1}^{i_{n}} \alpha_{n}^{k}\left(E f_{t_{n}^{k}}\right)\left(E f_{t}\right)=E h_{n} \cdot E f_{t} .
$$


So

$$
\begin{aligned}
\int_{A} f_{t} d P & =E\left(1_{A} f_{t}\right)=E\left(h f_{t}\right)=\lim _{n \rightarrow \infty} E\left(h_{n} f_{t}\right) \\
& =E f_{t} \lim _{n \rightarrow \infty} E h_{n}=E f_{t} \cdot E h=P(A) E f_{t}
\end{aligned}
$$

for all $t \in T^{*}$, where $\lambda\left(T^{*}\right)=1$.

Proposition 5.2. For every $E \in \mathcal{E}=\mathcal{T} \otimes \mathcal{A} \otimes \mathcal{B}$, the following properties hold:

(i) the mapping $t \mapsto\left(P \times \mu_{t}\right)\left(E_{t}\right)$ is $\mathcal{T}$-measurable;

(ii) for $\lambda$-a.e. $t \in T$, the set $H_{t}^{-1}\left(E_{t}\right)$ is $\mathcal{A}$-measurable, and $P\left(H_{t}^{-1}\left(E_{t}\right)\right)=$ $\left(P \times \mu_{t}\right)\left(E_{t}\right)$.

Proof. Let $\mathcal{D}$ be the collection of sets $E \in \mathcal{E}$ satisfying properties (i) and (ii).

First, we show that each measurable triple product set $E=S \times A \times B \in \mathcal{E}$ satisfies (i)-(ii), implying that $E \in \mathcal{D}$. Indeed:

(i) If $t \notin S$, then $E_{t}=\emptyset$ and $\left(P \times \mu_{t}\right)\left(E_{t}\right)=0$. On the other hand, if $t \in S$, then $E_{t}=A \times B$ and $\left(P \times \mu_{t}\right)\left(E_{t}\right)=P(A) \mu_{t}(B)$ for all $t \in T$. Hence, $\left(P \times \mu_{t}\right)\left(E_{t}\right)=$ $1_{S}(t) P(A) \mu_{t}(B)$ for all $t \in T$. Because $S \in \mathcal{T}$ and $t \mapsto \mu_{t}(B)$ is $\mathcal{T}$-measurable, so is $t \mapsto\left(P \times \mu_{t}\right)\left(E_{t}\right)$.

(ii) If $t \notin S$, then $E_{t}=\emptyset$, and $P\left(H_{t}^{-1}\left(E_{t}\right)\right)=0=\left(P \times \mu_{t}\right)\left(E_{t}\right)$. On the other hand, if $t \in S$, then $E_{t}=A \times B$, so $H_{t}^{-1}\left(E_{t}\right)=A \cap g_{t}^{-1}(B) \in \mathcal{A}$. In this case, applying Lemma 1 to the square-integrable and almost surely uncorrelated random variables $1_{g_{t}^{-1}(B)}(t \in T)$ implies that, for $\lambda$-a.e. $t \in S$, one has

$$
\begin{aligned}
P\left(H_{t}^{-1}\left(E_{t}\right)\right) & =P\left(A \cap g_{t}^{-1}(B)\right)=\int_{A} 1_{g_{t}^{-1}(B)} d P=P(A) \int_{\Omega} 1_{g_{t}^{-1}(B)} d P \\
& =P(A) \mu_{t}(B)=\left(P \times \mu_{t}\right)(A \times B)=\left(P \times \mu_{t}\right)\left(E_{t}\right) .
\end{aligned}
$$

It remains to verify that the family $\mathcal{D}$ is a Dynkin (or $\lambda$-) class in the sense that:

(a) $T \times \Omega \times X \in \mathcal{D}$;

(b) if $E, E^{\prime} \in \mathcal{D}$ with $E \supset E^{\prime}$, then $E \backslash E^{\prime} \in \mathcal{D}$;

(c) if $E^{n}$ is an increasing sequence of sets in $\mathcal{D}$, then $\bigcup_{n=1}^{\infty} E^{n} \in \mathcal{D}$.

Then we can apply Dynkin's $\pi-\lambda$ theorem to establish that $\mathcal{D}=\mathcal{E}=\mathcal{T} \otimes \mathcal{A} \otimes \mathcal{B}$, because the set of products of measurable sets is a $\pi$-system, i.e., closed under finite intersections (see [5], p. 44, and 8], p. 404). In fact:

(a) $T \times \Omega \times X \in \mathcal{D}$ as a triple product of measurable sets.

(b) If $E, E^{\prime}$ satisfy properties (i) and (ii) with $E \supset E^{\prime}$, then $\left(E \backslash E^{\prime}\right)_{t}=E_{t} \backslash E_{t}^{\prime}$ and so:

(i) the mapping

$$
t \mapsto\left(P \times \mu_{t}\right)\left(E \backslash E^{\prime}\right)_{t}=\left(P \times \mu_{t}\right)\left(E_{t}\right)-\left(P \times \mu_{t}\right)\left(E_{t}^{\prime}\right)
$$

is $\mathcal{T}$-measurable.

(ii) for $\lambda$-a.e. $t \in T$, the set $H^{-1}\left(\left(E \backslash E^{\prime}\right)_{t}\right)=H_{t}^{-1}\left(E_{t}\right) \backslash H_{t}^{-1}\left(E_{t}^{\prime}\right)$ is $\mathcal{A}$-measurable, with

$$
\begin{aligned}
P\left(H^{-1}\left(\left(E \backslash E^{\prime}\right)_{t}\right)\right) & =P\left(H_{t}^{-1}\left(E_{t}\right)\right)-P\left(H_{t}^{-1}\left(E_{t}^{\prime}\right)\right) \\
& =\left(P \times \mu_{t}\right)\left(E_{t}\right)-\left(P \times \mu_{t}\right)\left(E_{t}^{\prime}\right)=\left(P \times \mu_{t}\right)\left(\left(E \backslash E^{\prime}\right)_{t}\right) .
\end{aligned}
$$


Hence, $E \backslash E^{\prime} \in \mathcal{D}$.

(c) If $E^{n}$ is an increasing sequence in $\mathcal{D}$, then:

(i) the mapping

$$
t \mapsto\left(P \times \mu_{t}\right)\left(\bigcup_{n=1}^{\infty} E_{t}^{n}\right)=\lim _{n \rightarrow \infty}\left(P \times \mu_{t}\right)\left(E_{t}^{n}\right)
$$

is $\mathcal{T}$-measurable;

(ii) for $\lambda$-a.e. $t \in T$, the set $H_{t}^{-1}\left(\bigcup_{n=1}^{\infty} E_{t}^{n}\right)=\bigcup_{n=1}^{\infty} H_{t}^{-1}\left(E_{t}^{n}\right)$ is $\mathcal{A}$-measurable, and

$$
\begin{aligned}
P\left(H_{t}^{-1}\left(\bigcup_{n=1}^{\infty}\left(E_{t}^{n}\right)\right)\right. & =\lim _{n \rightarrow \infty} P\left(H_{t}^{-1}\left(E_{t}^{n}\right)\right)=\lim _{n \rightarrow \infty}\left(P \times \mu_{t}\right)\left(E_{t}^{n}\right) \\
& =\left(P \times \mu_{t}\right)\left(\bigcup_{n=1}^{\infty} E_{t}^{n}\right) .
\end{aligned}
$$

Hence, $\bigcup_{n=1}^{\infty} E^{n} \in \mathcal{D}$.

Proof of Theorem 3.1. Part (1) was proved as part of Proposition 5.2.

To prove part (2), note that given any $F \in \mathcal{F}$, there exists at least one $E \in \mathcal{E}$ such that $F=H^{-1}(E)$. Then $F_{t}=H_{t}^{-1}\left(E_{t}\right) \in \mathcal{A}$ for $\lambda$-a.e. $t \in T$, by Proposition 5.2. The same result implies that $P\left(F_{t}\right)=P\left(H_{t}^{-1}\left(E_{t}\right)\right)=\left(P \times \mu_{t}\right)\left(E_{t}\right)$, and that this is a $\mathcal{T}$-measurable function of $t$. So we can define a unique set function $\nu$ on the measurable space $(T \times \Omega, \mathcal{F})$ by $\nu(F):=\int_{T} P\left(F_{t}\right) d \lambda$. Note that $\nu(T \times \Omega)=1$ and, whenever $F^{n}(n=1,2 \ldots)$ is a disjoint countable collection of sets in $\mathcal{F}$, then

$$
\begin{aligned}
\nu\left(\bigcup_{n=1}^{\infty} F^{n}\right) & =\int_{T} P\left(\bigcup_{n=1}^{\infty} F_{t}^{n}\right) d \lambda=\int_{T} \sum_{n=1}^{\infty} P\left(F_{t}^{n}\right) d \lambda=\sum_{n=1}^{\infty} \int_{T} P\left(F_{t}^{n}\right) d \lambda \\
& =\sum_{n=1}^{\infty} \nu\left(F^{n}\right) .
\end{aligned}
$$

So $\nu$ is a uniquely defined probability measure.

To prove part (3), note first that whenever $F \in \mathcal{T} \otimes \mathcal{A}$, then $\nu(F)=\int_{T} P\left(F_{t}\right) d \lambda=$ $(\lambda \times P)(F)$. So $(T \times \Omega, \mathcal{F}, \nu)$ is an extension of $(T \times \Omega, \mathcal{T} \otimes \mathcal{A}, \lambda \times P)$. The remainder of the proof is virtually identical to that of the usual Fubini Theorem. For the sake of completeness, we include a proof adapted from [15] (p. 308). Let $V \subseteq L_{1}(T \times \Omega, \mathcal{F}, \nu)$ denote the set of all $\nu$-integrable functions $f$ that satisfy the one-way Fubini property $\int_{T \times \Omega} f d \nu=\int_{T}\left(\int_{\Omega} f_{t} d P\right) d \lambda$. Then $V$ includes every measurable indicator function $1_{F}(F \in \mathcal{F})$ because

$$
\nu(F)=\int_{T \times \Omega} 1_{F} d \nu=\int_{T} P\left(F_{t}\right) d \lambda=\int_{T}\left[\int_{\Omega}\left(1_{F}\right)_{t} d P\right] d \lambda .
$$

These equations confirm that the one-way Fubini property determines $\nu$ uniquely on $(T \times \Omega, \mathcal{F})$.

Next, $V$ is obviously closed under linear combinations; i.e., $V$ is a linear subspace. In particular, $V$ includes all measurable simple functions, and all differences between members of $V$. Also, any $\nu$-integrable function is the difference between two non-negative $\nu$ integrable functions, and any non-negative $\nu$-integrable function 
$f$ is the limit of an increasing sequence $f^{n}$ of non-negative simple functions. So it remains only to show that $V$ contains the integrable limit of an increasing sequence $f^{n}$ of non-negative functions in $V$.

Indeed, suppose $f \in L_{1}(T \times \Omega, \mathcal{F}, \nu)$ and let $f^{n}(n=1,2, \ldots)$ be any sequence of non-negative functions in $V$ satisfying $f^{n} \uparrow f$ as $n \rightarrow \infty$. Then the monotone convergence theorem implies that $\lim _{n \rightarrow \infty} \int_{T \times \Omega} f^{n} d \nu=\int_{T \times \Omega} f d \nu$. Since each $f^{n}$ is in $V$, and so satisfies the one-way Fubini property, we know that $f_{t}^{n}$ is in $L_{1}(\Omega, \mathcal{A}, P)$ for $\lambda$-a.e. $t \in T$. It is obvious that for $\lambda$-a.e. $t \in T, f_{t}^{n} \uparrow f_{t}$, and hence $f_{t}$ is $\mathcal{A}$-measurable with $\int_{\Omega} f_{t} d P=\lim _{n \rightarrow \infty} \int_{\Omega} f_{t}^{n} d P$. In fact, one must have $\int_{\Omega} f_{t}^{n} d P \uparrow \int_{\Omega} f_{t} d P$. Hence, the monotone convergence theorem and the one-way Fubini property for $f^{n}$ imply that

$$
\int_{T}\left(\int_{\Omega} f_{t} d P\right) d \lambda=\lim _{n \rightarrow \infty} \int_{T}\left(\int_{\Omega} f_{t}^{n} d P\right) d \lambda=\lim _{n \rightarrow \infty} \int_{T \times \Omega} f^{n} d \nu=\int_{T \times \Omega} f d \nu
$$

So $f$ also satisfies the one-way Fubini property.

This shows that $V=L_{1}(T \times \Omega, \mathcal{F}, \nu)$.

\section{REFERENCES}

1. Anderson, R.M. A nonstandard representation of Brownian motion and Itô-integration. Israel J. Math. 25 (1976), 15-46. MR0464380(57:4311)

2. Anderson, R.M. Nonstandard analysis with applications to economics. In Handbook of Mathematical Economics, Vol. IV (W. Hildenbrand and H. Sonnenschein, eds.). North-Holland, New York, 1991. MR1207198

3. Berger, J., Osswald, H., Sun, Y.N. and Wu, J.L. On nonstandard product measure spaces. Illinois J. Math. 46 (2002), 319-330. MR1936091 (2004g:28021)

4. Billingsley, P. Convergence of Probability Measures. Wiley, New York, 1968. MR0233396 $(38: 1718)$

5. Cohn, D.J. Measure Theory. Birkhäuser, Boston, 1980. MR0578344 (81k:28001)

6. Doob, J.L. Stochastic processes depending on a continuous parameter. Trans. Amer. Math. Soc. 42 (1937), 107-140. MR1501916

7. Doob, J.L. Stochastic Processes, Wiley, New York, 1953. MR0058896 (15:445b)

8. Durrett, R. Probability: Theory and Examples, Wadsworth, Belmont, California, 1991. MR:1068527 (91m:60002)

9. Hammond, P.J. and Sun, Y.N. Monte Carlo simulation of macroeconomic risk with a continuum of agents: the symmetric case. Economic Theory 21 (2003), 743-766. MR2014352

10. Judd, K.L. The law of large numbers with a continuum of iid random variables. J. Econ. Theory 35 (1985), 19-25. MR0786985 (86g:90025)

11. Keisler, H.J. Hyperfinite model theory. In Logic Colloquium 76 (R. O. Gandy and J. M. E. Hyland, eds.) North-Holland, Amsterdam, 1977. MR0491155 (58:10421)

12. Loeb, P.A. Conversion from nonstandard to standard measure spaces and applications in probability theory. Trans. Amer. Math. Soc. 211 (1975), 113-122. MR0390154 (52:10980)

13. Loeb, P.A. A nonstandard functional approach to Fubini's theorem. Proc. Amer. Math. Soc. 93 (1985), 343-346. MR0770550 (86f:28026)

14. Loeb, P.A. and Wolff, M.P.H. (eds.) Nonstandard Analysis for the Working Mathematician. Kluwer Academic Publishers, Dordrecht, 2000. MR.1790871 (2001e:03006)

15. Royden, H.L. Real Analysis, Macmillan, New York, 1968. MR0151555 (27:1540)

16. Sun, Y.N. Hyperfinite law of large numbers. Bull. Symbolic Logic 2 (1996), 189-198. MR 1396854 (97b:60054)

17. Sun, Y.N. A theory of hyperfinite processes: the complete removal of individual uncertainty via exact LLN. J. Math. Econ. 29 (1998), 419-503. MR1627287(99j:28020) 
18. Sun, Y.N. The almost equivalence of pairwise and mutual independence and the duality with exchangeability. Probability Theory and Related Fields. 112 (1998), 425-456. MR.1660898 (2000m:60002)

19. Sun, Y.N. The exact law of large numbers via Fubini extension and the characterization of insurable risks, J. Econ. Theory, published online, January 2005.

Department of Economics, Stanford University, 579 Serra Mall, Stanford, CaliforNIA $94305-6072$

E-mail address: peter.hammond@stanford.edu

Department of Mathematics, National University of Singapore, 2 Science Drive 2, Singapore 117543 - And - Department of Economics, National University of Singapore, 1 ARTs Link, Singapore 117570

E-mail address: matsuny@nus.edu.sg 